Tom 26 № 4

\title{
ИНТЕРПОЛЯЦИОННО-ОРТОГОНАЛЬНЫЕ БАЗИСЫ КМА И ВСПЛЕСКОВ ${ }^{1}$
}

\section{Е. А. Плещева}

\begin{abstract}
Основной целью данной статьи является построение ортонормированных базисов кратномасштабного анализа (KMA), которые при этом являются интерполяционными на сетке $k / 2^{j}$. Рассматриваются ортонормированный KMA и соответствующие всплески. На основе такого КМА по ортогональным маскам масштабирующих функций строятся маски новых масштабирующих функций, удовлетворяющие условию интерполяционности. В книге Добеши (2001) показано, что одновременно интерполяционные и ортогональные базисы KMА не могут иметь компактный носитель. В работе 2008 г. Ю. Н. Субботин и Н. И. Черных привели способ модификации масштабирующей функции Мейера таким образом, чтобы образованный ею базис был одновременно ортогональным и интерполяционным. В данной статье получен способ модификации более широкого класса масштабирующих функций таким образом, чтобы новые масштабирующие функции, оставаясь ортогональными, стали еще и интерполяционными, начиная построение с маски масштабирующей функции. Сформулированы необходимые и достатоточные условия для того, чтобы сдвиги вновь полученной с использованием модифицированной маски масштабирующей функции образовывали интерполяционно-ортогональную систему.
\end{abstract}

Ключевые слова: ортогональный всплеск, интерполяционный всплеск, масштабирующая функция, базис, кратномасштабный анализ, маска масштабирующей функции.

\section{E. A. Pleshcheva. Interpolating orthogonal bases of an MRA and wavelets.}

The main goal of this paper is to construct orthonormal bases of a multiresolution analysis (MRA) that are interpolating on the grid $k / 2^{j}$. We consider an orthonormal MRA and the corresponding wavelets. Based on this MRA and using orthogonal masks of the scaling functions, we construct new masks of scaling functions that satisfy the interpolation condition. In I. Daubechies's book it is proved that bases of an MRA that are interpolating and orthogonal simultaneously cannot have a compact support. In 2008, Yu.N. Subbotin and N.I. Chernykh suggested a method for modifying the Meyer scaling function in such a way that the basis formed by it is simultaneously orthogonal and interpolating. In the present paper we propose a method for modifying a wider class of scaling functions in such a way that the new scaling functions remain orthogonal and at the same time become interpolating. We start the construction with a mask of a scaling function and find necessary and sufficient conditions for the shifts of the scaling function obtained with the use of the modified mask to form an interpolating orthogonal system.

Keywords: orthogonal wavelet, interpolating wavelet, scaling function, basis, multiresolution analysis, mask of scaling function.

MSC: $42 \mathrm{C} 40$

DOI: $10.21538 / 0134-4889-2020-26-4-224-233$

\section{Введение}

Классическая теория всплесков стала интенсивно развиваться в 80-е годы прошлого века. После работ Мейера и Малла (см. [1;2]) построение базисов всплесков начинается с построения системы вложенных подпространств $V_{j}$ пространства $L^{2}(\mathbb{R})$, называемой кратномасштабным анализом пространства $L^{2}(\mathbb{R})$.

Известно следующее определение.

О п р е д е л е н и е. Последовательность вложенных друг в друга замкнутых подпространств пространства $L^{2}(\mathbb{R})$

$$
\ldots \subset V_{-1} \subset V_{0} \subset V_{1} \subset V_{2} \subset \cdots
$$

\footnotetext{
${ }^{1}$ Работа выполнена в рамках исследований, проводимых в Уральском математическом центре.
} 
называется кратномасштабным анализом (KMA) пространства $L^{2}(\mathbb{R})$, если она удовлетворяет следующим условиям:

a) $\overline{\cup_{j} V_{j}}=L^{2}(\mathbb{R})$

b) $\cap_{j} V_{j}=\{0\}$;

c) $f(x) \in V_{j} \Leftrightarrow f\left(x+l / 2^{j}\right) \in V_{j} \quad \forall j, l \in \mathbb{Z}$;

d) $f(x) \in V_{0} \Leftrightarrow f\left(2^{j} x\right) \in V_{j} \quad \forall j \in \mathbb{Z}$

е) найдется такая функция $\varphi(x) \in L^{2}(\mathbb{R})$, что множество ее сдвигов $\{\varphi(x+k)\}_{k \in \mathbb{Z}}$ образует ортонормированный базис пространств $V_{0}$.

Базис $\left\{\varphi_{j, k}(x):=2^{j / 2} \varphi\left(2^{j} x-k\right)\right\}_{k \in \mathbb{Z}}$ каждого из пространств $V_{j}$ образован сжатиями и сдвигами масштабирующей функции $\varphi(x) \in L^{2}(\mathbb{R})$. Далее строятся такие подпространства $W_{j}$, ортогональные базисы которых $\left\{\psi_{j, k}(x)\right\}_{k \in \mathbb{Z}}$ образованы также сдвигами и сжатиями одной функции, что $W_{j}$ является ортогональным дополнением пространства $V_{j}$ до $V_{j+1}$. Полученная в результате система $\left\{\psi_{j, k}(x)\right\}_{j, k \in \mathbb{Z}}$ образует ортонормированный базис всего пространства $L^{2}(\mathbb{R})$.

Хорошо известно также, что для того чтобы система $\left\{\varphi_{j, k}(x):=2^{j / 2} \varphi\left(2^{j} x-k\right)\right\}_{k \in \mathbb{Z}}$ была ортонормированной, а система $\left\{2^{-j / 2} \varphi_{j, k}(x)\right\}_{k \in \mathbb{Z}}$ - интерполяционной, необходимо и достаточно, чтобы выполнялись два условия:

$$
\sum_{\nu \in \mathbb{Z}}|\widehat{\varphi}(\omega-k)|^{2} \stackrel{\text { п. в. }}{=} 1, \quad \sum_{\nu \in \mathbb{Z}} \widehat{\varphi}(\omega-k) \stackrel{\text { п.в. }}{=} 1 .
$$

В статье [3] Ю.Н. Субботин и Н. И. Черных показали, как можно изменить вещественнозначную масштабирующую функцию типа Мейера, чтобы новая масштабирующая функция, оставаясь ортогональной, стала еще и интерполяционной. Рассмотрим функцию $\widehat{\varphi}(\omega)=\widehat{\varphi_{\varepsilon}}(\omega)$, носитель которой совпадает с отрезком

$$
[-(1+\varepsilon) / 2,(1+\varepsilon) / 2], \quad 0 \leq \varepsilon \leq 1 / 3,
$$

$\widehat{\varphi}(\omega)=1$ при $-(1-\varepsilon) / 2 \leq \omega \leq(1-\varepsilon) / 2 ; \widehat{\varphi}(\omega)$ четная, и на промежутке $[(1-\varepsilon) / 2,(1+$ $\varepsilon) / 2$ ] функция $\widehat{\varphi}^{2}(\omega)-1 / 2$ нечетная относительно $w=1 / 2$. Для такой функции выполнено условие ортонормированности. В работе [3] предложено два способа построения функций на основе описанных функций типа Мейера, таких, что новая масштабирующая функция будет порождать ортонормированную и интерполяционную систему.

При первом способе

$$
\widehat{\varphi}_{1}(\omega)=\widehat{\varphi}(\omega)+\alpha(\omega)+i \cdot \operatorname{sign}(\omega) \beta(\omega),
$$

где носитель функций $\alpha(\omega), \beta(\omega)$ - это множество $[(1-\varepsilon) / 2,(1+\varepsilon) / 2] \cup[(-1-\varepsilon) / 2,(-1+\varepsilon) / 2]$, а на промежутках $[(1-\varepsilon) / 2,(1+\varepsilon) / 2]$ и $[(-1-\varepsilon) / 2,(-1+\varepsilon) / 2]$

$$
\alpha(\omega)=\frac{1-\widehat{\varphi}(\omega)-\widehat{\varphi}(\omega-1)-\widehat{\varphi}(\omega+1)}{2}, \quad \beta(\omega)=\sqrt{\frac{\widehat{\varphi}(\omega)(\widehat{\varphi}(\omega-1)+\widehat{\varphi}(\omega+1))}{2}} .
$$

При втором способе

$$
\widehat{\varphi}_{2}(\omega)=|\widehat{\varphi}(\omega)|^{2}+i \cdot \operatorname{sign}(\omega) \beta(\omega),
$$

где носитель $\beta(\omega)$ - также множество $[(1-\varepsilon) / 2,(1+\varepsilon) / 2] \cup[(-1-\varepsilon) / 2,(-1+\varepsilon) / 2]$, а на своем носителе

$$
\beta(\omega)=\widehat{\varphi}(\omega)(\widehat{\varphi}(\omega-1)+\widehat{\varphi}(\omega+1)) .
$$

В обоих случаях новая функция $\varphi^{s}(s=1,2)$ удовлетворяет условиям ортогональности и интерполяционности и является масштабирующей функцией для кратномасштабного анализа $\ldots \subset V_{j} \subset V_{j+1} \subset \ldots$, где базис пространства $V_{0}$ образован системой $\left\{\varphi^{s}(x-k)\right\}_{k \in \mathbb{Z}}(s=1,2)$.

Условия вложения (1) выполняются при выполнении масштабирующего соотношения

$$
\varphi(x)=\sum_{\nu \in \mathbb{Z}} h_{\nu} \varphi_{1, \nu}(x), \quad\left\{h_{\nu}\right\} \in l^{2}(\mathbb{Z}) .
$$


Для пространств $V_{j}$ строятся подпространства $W_{j}$, дополняющие их до следующего пространства $V_{j+1}$ таким образом, что выполняются условия

1) $V_{j} \oplus W_{j}=V_{j+1}$

2) $V_{j} \perp W_{j} \quad \forall j \in \mathbb{Z}$.

Базисы пространств $W_{j}$ образованы функциями-всплесками $\left\{\psi_{j, k}(x)=2^{j / 2} \psi\left(2^{j} x-k\right)\right\}_{k \in \mathbb{Z}}$, где функция $\psi$ строится по $\varphi$ следующим образом:

$$
\psi(x)=\sum_{\nu \in \mathbb{Z}}(-1)^{\nu-1} \overline{h_{1-\nu}} \varphi_{1, \nu}(x),
$$

где $h_{\nu}$ - коэффициенты из $(3), \varphi_{j, \nu}(x)=2^{j / 2} \varphi\left(2^{j} x-\nu\right)$.

В данной работе получен способ модификации любых, а не только мейеровских, масштабирующих функций, образующих ортогональный KMA, таким образом, чтобы новые масштабирующие функции порождали интерполяционно-ортогональные базисы.

\section{1. Необходимые условия интерполяционности в терминах масок масштабирующей функции подпространств $\mathrm{KMA}$ пространства $L^{2}(\mathbb{R})$}

Известно, что необходимым и достаточным условием ортонормированности целочисленных сдвигов масштабирующей функции любого КМА является условие

$$
\sum_{\nu \in \mathbb{Z}}|\widehat{\varphi}(\omega-\nu)| \stackrel{\text { ח.в. }}{=} 1
$$

(п. в. - почти всюду). Известно также, что необходимое и достаточное условие интерполяцион-

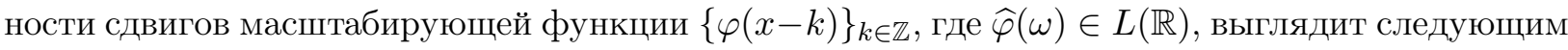
образом:

$$
\sum_{\nu \in \mathbb{Z}} \widehat{\varphi}(\omega-\nu) \stackrel{\text { п.в. }}{=} 1
$$

Масштабирующие соотношения (3) после преобразования Фурье можно представить в виде

$$
\widehat{\varphi}(\omega)=m\left(\frac{\omega}{2}\right) \widehat{\varphi}\left(\frac{\omega}{2}\right),
$$

где 1-периодическая функция $m(\omega)$ называется маской масштабирующей функиии и определяется формулой

$$
m(\omega)=\frac{1}{\sqrt{2}} \sum_{\nu \in \mathbb{Z}} h_{\nu} e^{2 \pi i \nu \omega} \in L^{2}[0,1] .
$$

Известно, что если система $\{\varphi(x-k)\}_{k \in \mathbb{Z}}$ ортонормирована, то для маски выполнено соотношение

$$
|m(\omega)|^{2}+\left|m\left(\omega+\frac{1}{2}\right)\right|^{2} \stackrel{\text { п. В. }}{=} 1 .
$$

Докажем, что подобное утверждение справедливо и для условия интерполяционности.

Предложение. Пусть система иелочисленных сдвигов масштабирующей функиии $\{\varphi(x-k)\}_{k \in \mathbb{Z}}$ является интерполяционной, а $\widehat{\varphi}(\omega) \in L(\mathbb{R})$. Тогда для маски масштабирующей функции $m(\omega)$ выполняется условие

$$
m(\omega)+m\left(\omega+\frac{1}{2}\right) \stackrel{\text { п.в. }}{=} 1 .
$$

Д о к а з а т е л ь с т в о. Пусть система $\{\varphi(x-k)\}_{k \in \mathbb{Z}}$ является интерполяционной. Тогда для функции $\varphi(x)$ выполняется условие (4). Стандартным образом, подставляя (5) в (4) и 
разбивая получившуюся сумму на суммы по четным и нечетным слагаемым, получаем, что справедливо равенство

$$
\begin{gathered}
\text { 1п. в. }=\sum_{\nu \in \mathbb{Z}} \hat{\varphi}(\omega-k)=\sum_{\nu \in \mathbb{Z}} m\left(\frac{\omega-k}{2}\right) \hat{\varphi}\left(\frac{\omega-k}{2}\right) \\
=\sum_{\nu \in \mathbb{Z}} m\left(\frac{\omega}{2}-k\right) \hat{\varphi}\left(\frac{\omega}{2}-k\right)+\sum_{\nu \in \mathbb{Z}} m\left(\frac{\omega+1}{2}-k\right) \hat{\varphi}\left(\frac{\omega+1}{2}-k\right) .
\end{gathered}
$$

В силу 1-периодичности $m(\omega)$ и равенства (4) для почти всех $\omega$ имеем

$$
m\left(\frac{\omega}{2}\right) \sum_{\nu \in \mathbb{Z}} \widehat{\varphi}\left(\frac{\omega}{2}-k\right)+m\left(\frac{\omega+1}{2}\right) \sum_{\nu \in \mathbb{Z}} \widehat{\varphi}\left(\frac{\omega+1}{2}-k\right)=m\left(\frac{\omega}{2}\right)+m\left(\frac{\omega}{2}+\frac{1}{2}\right) \text { п. в. }=1 .
$$

Таким образом, доказана справедливость (8).

\section{2. Преобразование маски масштабирующей функции}

Пусть имеется ортонормированный КМА с маской $m(\omega)$, принимающей вещественные значения. Для него справедливы масштабирующие соотношения (3), (5) и определена маска равенством (6). Преобразуем маску масштабирующей функции следующим образом:

$$
m_{I}(\omega)=|m(\omega)|^{2}+\alpha(\omega) .
$$

Подберем такую 1-периодическую $\alpha(\omega)$, чтобы новая маска удовлетворяла условию (8). Для этого должно выполняться равенство $\alpha(\omega)=-\alpha(\omega+1 / 2)$. При этом, чтобы для $m_{I}(\omega)$, как и для исходной $m(\omega)$, выполнялось условие типа $(7)$, определим $\alpha(\omega)$ в виде

$$
\alpha(\omega)=B(\omega) m(\omega) m\left(\omega+\frac{1}{2}\right), \quad \text { где } \quad B(\omega) \stackrel{\text { п.в. }}{=}-B\left(\omega+\frac{1}{2}\right),
$$

и наложим на $B(\omega)$ условия

$$
B(\omega) \stackrel{\text { ח.в. }}{=}-\overline{B(\omega)}, \quad|B(\omega)| \stackrel{\text { п.в. }}{=} 1 .
$$

Этим условиям удовлетворяет, например,

$$
B(\omega)=i \cdot \operatorname{sign}(\sin 2 \pi \omega) .
$$

Таким образом, новая маска, по которой будем строить модифицированный KMA, теперь имеет вид

$$
m_{I}(\omega)=|m(\omega)|^{2}+i \cdot \operatorname{sign}(\sin 2 \pi \omega) m(\omega) m\left(\omega+\frac{1}{2}\right) .
$$

В более общем случае, включая комплекснозначные $m(\omega)$, можно преобразовать маску, положив

$$
m_{I}(\omega)=|m(\omega)|^{2}+i \cdot \operatorname{sign}(\sin 2 \pi \omega)|m(\omega)|\left|m\left(\omega+\frac{1}{2}\right)\right| .
$$

Легко проверить, что условие (9) для вещественнозначных масок и условие (10) для комплекснозначных масок обеспечивают выполнение равенств (7), (8). Докажем, что при дополнительных условиях на маски $m(\omega)$ масштабирующую функцию, порождающую интерполяционно-ортогональную систему, можно восстановить по ее преобразованию Фурье

$$
\widehat{\varphi_{I}}(\omega)=\prod_{j=1}^{\infty} m_{I}\left(\frac{\omega}{2^{j}}\right) .
$$

Справедлива следующая теорема. 
Теорема 1. Пусть маска $m(\omega)$ удовлетворяет условиям

1) $|m(\omega)|^{2}+|m(\omega+1 / 2)|^{2}=1$

2) $|m(\omega)| \geq C>0$ npu $|\omega| \leq 1 / 4$;

3) функция $|m(\omega+1 / 2)| \leq \Omega(\omega)$ при $|\omega|<\delta_{0}$, где $\Omega(\omega)$ непрерывна, а рлд $\sum_{j=1}^{\infty} \Omega\left(\omega / 2^{j}\right)$ сходится.

Пусть, кроме того, $m(0)=1, m(\omega)$ непрерывна в окрестности нуля $u$

$$
\prod_{j=1}^{\infty} m\left(\omega / 2^{j}\right)=: \widehat{\varphi}(\omega) \in L(\mathbb{R})
$$

функиия $m_{I}(\omega)$ определяется формулой $(9)$ при $m(\omega)$ со значениями из $\mathbb{R}$ или формулой (10) при $m(\omega)$, принимающей любые значения, в том числе и комплексные.

Тогда при цельх $ј$ система функиий $\left\{\varphi_{I, j, k}\right\}_{k \in \mathbb{Z}}$, где $\widehat{\varphi_{I}}$ определена формулой (11), является ортонормированной в пространстве $L^{2}(\mathbb{R})$, а система $\left\{2^{-j / 2} \varphi_{I, j, k}\right\}_{k \in \mathbb{Z}}-$ интерполяи, онной на сетке $\left\{x_{j, l}=l / 2^{j}: l \in \mathbb{Z}\right\}$. Последовательность пространств

$$
V_{j}^{I}:=\operatorname{span}\left\{\varphi_{I, j, k}\right\}_{k \in \mathbb{Z}}
$$

образует KMA пространства $L^{2}(\mathbb{R})$.

Д о к а з а т е л ь с т в о. Заметим, что

$$
\left|m_{I}(\omega)\right|^{2}=|m(\omega)|^{4}+|m(\omega)|^{2}|m(\omega+1 / 2)|^{2}=|m(\omega)|^{2}\left(|m(\omega)|^{2}+|m(\omega+1 / 2)|^{2}\right)=|m(\omega)|^{2} .
$$

Для бесконечного произведения $\prod_{j=1}^{\infty} m_{I}\left(\omega / 2^{j}\right)$ справедливо равенство

$$
\prod_{j=1}^{\infty} m_{I}\left(\frac{\omega}{2^{j}}\right)=\prod_{j=1}^{\infty} e^{\ln m_{I}\left(\omega / 2^{j}\right)}=e^{\sum_{j=1}^{\infty} \ln m_{I}\left(\omega / 2^{j}\right)} .
$$

Ряд $\sum_{j=1}^{\infty} \ln m_{I}\left(\omega / 2^{j}\right)$ сходится, если абсолютно сходится ряд $\sum_{j=1}^{\infty}\left(m_{I}\left(\omega / 2^{j}\right)-1\right)$. Так как для $m(\omega)$ выполнено условие 3), то

$$
\sum_{j=1}^{\infty}\left|m_{I}\left(\frac{\omega}{2^{j}}\right)-1\right|=\sum_{j=1}^{\infty}\left|m_{I}\left(\frac{\omega}{2^{j}}+\frac{1}{2}\right)\right|=\sum_{j=1}^{\infty}\left|m\left(\frac{\omega}{2^{j}}+\frac{1}{2}\right)\right|<\infty .
$$

Тогда и бесконечное произведение $\prod_{j=1}^{\infty} m_{I}\left(\omega / 2^{j}\right)$ сходится.

Следовательно, определив в (11) функцию $\widehat{\varphi_{I}}(\omega):=\prod_{j=1}^{\infty} m_{I}\left(\omega / 2^{j}\right)$, получим, что ее модуль совпадает с модулем исходной масштабирующей функции, т. е.

$$
\left|m_{I}(\omega)\right|=|m(\omega)|, \quad|\widehat{\varphi}(\omega)|=|\widehat{\varphi}(\omega)| .
$$

По условию теоремы $|\widehat{\varphi}(\omega)| \in L(\mathbb{R})$, а так как $m(\omega)$ удовлетворяет достаточному условию ортогональности масштабирующих функций, то

$$
\prod_{j=1}^{\infty} m_{I}\left(\frac{\omega}{2^{j}}\right) \in L(\mathbb{R}) \cap L^{2}(\mathbb{R}) \Leftrightarrow \prod_{j=1}^{\infty} m\left(\frac{\omega}{2^{j}}\right) \in L(\mathbb{R}) \cap L^{2}(\mathbb{R}) .
$$


Введем последовательность функций

$$
\widehat{\varphi_{I}^{n}}(\omega):=\prod_{j=1}^{n} m_{I}\left(\frac{\omega}{2^{j}}\right) \chi_{\left[-\frac{1}{2}, \frac{1}{2}\right]}\left(\frac{\omega}{2^{n}}\right)
$$

Очевидно, $\widehat{\varphi_{I}^{n}} \underset{n \rightarrow \infty}{\rightarrow} \widehat{\varphi_{I}}$ поточечно.

Покажем, что системы $\left\{\varphi_{I}^{n}(x-k)\right\}_{k \in \mathbb{Z}}$ являются интерполяционно-ортогональными.

По построению $m_{I}(\omega)$ удовлетворяет условию (8). Покажем, что $\left\{\varphi_{I}^{n}(x-k)\right\}_{k \in \mathbb{Z}}$ являются интерполяционными на целочисленной сетке системы. Имеем

$$
\varphi_{I}^{n}(k)=\int_{\mathbb{R}} \widehat{\varphi_{I}^{n}}(\omega) e^{2 \pi i k \omega} d \omega=\int_{-2^{n-1}}^{2^{n-1}} \prod_{j=1}^{n} m_{I}\left(\frac{\omega}{2^{j}}\right) e^{2 \pi i k \omega} d \omega .
$$

Отрезок интегрирования в данном случае совпадает с периодом подынтегральной функции. Сдвигая отрезок интегрирования на $2^{n-1}$ и разбивая на сумму двух интегралов, получим

$$
\varphi_{I}^{n}(k)=\int_{0}^{2^{n}} \prod_{j=1}^{n} m_{I}\left(\frac{\omega}{2^{j}}\right) e^{2 \pi i k \omega} d \omega=\int_{0}^{2^{n-1}} \prod_{j=1}^{n} m_{I}\left(\frac{\omega}{2^{j}}\right) e^{2 \pi i k \omega} d \omega+\int_{2^{n-1}}^{2^{n}} \prod_{j=1}^{n} m_{I}\left(\frac{\omega}{2^{j}}\right) e^{2 \pi i k \omega} d \omega,
$$

где после замены во втором интеграле переменной $\omega / 2^{n}$ на $\omega / 2^{n}+1 / 2$ имеем

$$
\varphi_{I}^{n}(k)=\int_{0}^{2^{n-1}} \prod_{j=1}^{n-1} m_{I}\left(\frac{\omega}{2^{j}}\right)\left(m_{I}\left(\frac{\omega}{2^{n}}\right)+m_{I}\left(\frac{\omega}{2^{n}}+\frac{1}{2}\right)\right) e^{2 \pi i k \omega} d \omega=\int_{0}^{2^{n-1}} \prod_{j=1}^{n-1} m_{I}\left(\frac{\omega}{2^{j}}\right) e^{2 \pi i k \omega} d \omega .
$$

Заметим, что под знаком интеграла теперь оказалась функция $\widehat{\varphi_{I}^{n-1}}(\omega)$, т. е. исходное равенство после $n$ итераций примет вид

$$
\begin{gathered}
\varphi_{I}^{n}(k)=\int_{\mathbb{R}} \widehat{\varphi_{I}^{n-1}}(\omega) e^{2 \pi i k \omega} d \omega=\ldots=\int_{\mathbb{R}} \widehat{\varphi_{I}^{n-1}}(\omega) e^{2 \pi i k \omega} d \omega=\int_{-1}^{1} m_{I}\left(\frac{\omega}{2}\right) e^{2 \pi i k \omega} d \omega \\
=\int_{0}^{1} m_{I}\left(\frac{\omega}{2}\right) e^{2 \pi i k \omega} d \omega+\int_{1}^{2} m_{I}\left(\frac{\omega}{2}\right) e^{2 \pi i k \omega} d \omega=\int_{0}^{1}\left(m_{I}\left(\frac{\omega}{2}\right)+m_{I}\left(\frac{\omega}{2}+\frac{1}{2}\right)\right) e^{2 \pi i k \omega} d \omega \\
=\int_{0}^{1} e^{2 \pi i k \omega} d \omega=\delta_{k, 0} .
\end{gathered}
$$

Таким образом, интерполяционность систем $\left\{\varphi_{I}^{n}(x-k)\right\}_{k \in \mathbb{Z}}$ доказана.

Аналогично

$$
\begin{aligned}
\left\langle\varphi_{I}^{n}(x), \varphi_{I}^{n}(x-k)\right\rangle_{L^{2}(\mathbb{R})}=\int_{\mathbb{R}} \widehat{\varphi_{I}^{n}}(\omega) \overline{\varphi_{I}^{n}}(\omega) e^{-2 \pi i k \omega} d \omega=\int_{-2^{n-1}}^{2^{n-1}} \prod_{j=1}^{n}\left|m_{I}\left(\frac{\omega}{2^{j}}\right)\right|^{2} e^{2 \pi i k \omega} d \omega \\
=\int_{0}^{2_{j=1}^{n}} \prod_{j}^{n}\left|m_{I}\left(\frac{\omega}{2^{j}}\right)\right|^{2} e^{2 \pi i k \omega} d \omega=\int_{0}^{2^{n-1}} \prod_{j=1}^{n}\left|m_{I}\left(\frac{\omega}{2^{j}}\right)\right|^{2} e^{2 \pi i k \omega} d \omega+\int_{2^{n-1}}^{2^{n}} \prod_{j=1}^{n}\left|m_{I}\left(\frac{\omega}{2^{j}}\right)\right|^{2} e^{2 \pi i k \omega} d \omega
\end{aligned}
$$




$$
\begin{gathered}
=\int_{0}^{2^{n-1}} \prod_{j=1}^{n-1}\left|m_{I}\left(\frac{\omega}{2^{j}}\right)\right|^{2}\left(\left|m_{I}\left(\frac{\omega}{2^{n}}\right)\right|^{2}+\left|m_{I}\left(\frac{\omega}{2^{n}}+\frac{1}{2}\right)\right|^{2}\right) e^{2 \pi i k \omega} d \omega=\int_{0}^{2^{n-1}} \prod_{j=1}^{n-1}\left|m_{I}\left(\frac{\omega}{2^{j}}\right)\right|^{2} e^{2 \pi i k \omega} d \omega \\
=\int_{\mathbb{R}}\left|\widehat{\varphi_{I}^{n-1}}(\omega)\right|^{2} e^{2 \pi i k \omega} d \omega=\ldots=\int_{\mathbb{R}}\left|\widehat{\varphi_{I}^{n-1}}(\omega)\right|^{2} e^{2 \pi i k \omega} d \omega=\int_{-1}^{1}\left|m_{I}\left(\frac{\omega}{2}\right)\right|^{2} e^{2 \pi i k \omega} d \omega \\
=\int_{0}^{1}\left|m_{I}\left(\frac{\omega}{2}\right)\right|^{2} e^{2 \pi i k \omega} d \omega+\int_{1}^{2}\left|m_{I}\left(\frac{\omega}{2}\right)\right|^{2} e^{2 \pi i k \omega} d \omega=\int_{0}^{1}\left(\left|m\left(\frac{\omega}{2}\right)\right|^{2}+\left|m\left(\frac{\omega}{2}+\frac{1}{2}\right)\right|^{2}\right) e^{2 \pi i k \omega} d \omega \\
=\int_{0}^{1} e^{2 \pi i k \omega} d \omega=\delta_{k, 0} .
\end{gathered}
$$

Следовательно, системы $\left\{\varphi_{I}^{n}(x-k)\right\}_{k \in \mathbb{Z}}$ являются также ортонормированными.

Покажем теперь, что

$$
\left|\widehat{\varphi_{I}^{n}}(\omega)\right| \leq C_{1}\left|\widehat{\varphi_{I}}(\omega)\right| .
$$

Тогда по теореме Лебега о мажорантной сходимости можно будет перейти к пределам под знаками интегралов. По условию (12) $\left|m\left(\omega / 2^{j}\right)\right|=\left|m_{I}\left(\omega / 2^{j}\right)\right|$, поэтому из условия 2) теоремы следует, что $\left|m_{I}\left(\omega / 2^{j}\right)\right| \geq C>0$ при $|\omega| \leq 1 / 2, j \geq 1$. При этом $|m(\omega)-1| \leq \Omega(\omega)$ при $\omega<\delta_{0}$. Отсюда следует, что $|m(\omega)|=\left|m_{I}(\omega)\right| \geq 1-\Omega(\omega)$ при $\omega<\delta_{0}$. Найдется такое $j_{0}$, что $\Omega\left(\omega / 2^{j}\right)<1 / 2, j \geq j_{0}$. Имеем

$$
\left|\widehat{\varphi_{I}}(\omega)\right|=\prod_{j=1}^{\infty}\left|m_{I}\left(\frac{\omega}{2^{j}}\right)\right|=\prod_{j=1}^{j_{0}}\left|m_{I}\left(\frac{\omega}{2^{j}}\right)\right| \prod_{j=j_{0}+1}^{\infty}\left|m_{I}\left(\frac{\omega}{2^{j}}\right)\right| .
$$

Так как выполнены условия 2) теоремы, то

$$
\prod_{j=1}^{j_{0}}\left|m_{I}\left(\omega / 2^{j}\right)\right| \geq C^{j_{0}}
$$

По условию 3$)|m(\omega+1 / 2)| \leq \Omega(\omega)$, следовательно, $\left|m_{I}(\omega+1 / 2)\right|=\left|1-m_{I}(\omega)\right| \leq \Omega(\omega)$, поэтому $1-\left|m_{I}(\omega)\right| \leq \Omega(\omega)$ при $\omega<\delta_{0}$. Тогда

$$
\prod_{j=j_{0}+1}^{\infty}\left|m_{I}\left(\omega / 2^{j}\right)\right| \geq \prod_{j=j_{0}+1}^{\infty}\left(1-\Omega\left(2^{-j} \omega\right)\right) .
$$

Следовательно, используя то, что при $0 \leq y \leq 1 / 2$ выполнено $1-y \geq e^{-2 y}$, имеем

$$
\left|\widehat{\varphi_{I}}(\omega)\right| \geq C^{j_{0}} \prod_{j=j_{0}+1}^{\infty} e^{-2 \Omega\left(2^{-j} \omega\right)} \geq C^{j_{0}} e^{\sum_{j_{0}+1}^{\infty}\left(-2 \Omega\left(2^{-j} \omega\right)\right)} \geq C^{j_{0}} e^{-2 \max _{|\omega|<\delta_{0}} \Omega\left(2^{-j} \omega\right)}=C^{\prime}>0 .
$$

Это означает, что

$$
\chi_{\left[-\frac{1}{2}, \frac{1}{2}\right]}(\omega) \leq \frac{\left|\widehat{\varphi_{I}}(\omega)\right|}{C^{\prime}} .
$$

Тогда

$$
\left|\widehat{\varphi_{I}^{n}}(\omega)\right|=\prod_{j=1}^{n}\left|m_{I}\left(\frac{\omega}{2^{j}}\right)\right| \chi_{\left[\frac{1}{2}, \frac{1}{2}\right]}\left(\frac{\omega}{2^{n}}\right) \leq \frac{\prod_{j=1}^{n}\left|m_{I}\left(\omega / 2^{j}\right)\right|\left|\widehat{\varphi_{I}}\left(\omega / 2^{n}\right)\right|}{C^{\prime}}=C_{1}\left|\widehat{\varphi_{I}}(\omega)\right| \in L(\mathbb{R}) \cap L^{2}(\mathbb{R}) .
$$

Применив теорему Лебега о мажорантной сходимости, получаем, что выполнено

$$
\varphi_{I}(k)=\delta_{k, 0} ; \quad\left\langle\varphi_{I}(x), \varphi_{I}(x-k)\right\rangle_{L^{2}(\mathbb{R})}=\delta_{k, 0} .
$$




\section{3. Пространства всплесков}

Пространства $W_{I, j}$, которые являются ортогональными дополнениями пространств $V_{I, j}$ до $V_{I, j+1}$, строятся таким образом, что их базисы образованы системами $\left\{\psi_{I, j, k}(x)\right\}_{k \in \mathbb{Z}}$, где

$$
\widehat{\psi_{I}}(\omega)=e^{i \pi \omega} \overline{m_{I}\left(\frac{\omega+1}{2}\right)} \widehat{\varphi_{I}}\left(\frac{\omega}{2}\right)
$$

Тогда функция $\psi_{I}(x)=\sum_{\nu \in \mathbb{Z}}(-1)^{\nu-1} \bar{h}_{I, 1-\nu} \varphi_{I, 1, \nu}(x)$.

Масштабирующие соотношения для функций $\varphi_{I}$ выглядят следующим образом:

$$
\varphi_{I}(x)=\sum_{\nu \in \mathbb{Z}} h_{I, \nu} \varphi_{I, 1, \nu}(x) .
$$

Так как $\varphi_{I}(k)=\delta_{0, k}$, то выполняется

$$
\varphi_{I}(k)=\sum_{\nu \in \mathbb{Z}} h_{I, \nu} \varphi_{I, 1, \nu}(k)=\sum_{\nu \in \mathbb{Z}} h_{I, \nu} \sqrt{2} \varphi_{I}(2 k-\nu)=\sqrt{2} h_{2 k} \varphi(0) .
$$

Поэтому $h_{0}=1 / \sqrt{2}, h_{2 k}=0$. Отсюда следует, что справедлива следующая теорема.

Теорема 2. Пусть функиия $\psi_{I}(x)$ определена формулой (13). Тогда система функиий $\left\{\psi_{I, j, k}\right\}_{j, k \in \mathbb{Z}}-$ ортонормированный базис пространства $L^{2}(\mathbb{R})$, а система $\left\{2^{-j / 2} \psi_{I, j, k}\right\}_{j, k \in \mathbb{Z}}$ является интерполяиионной на сетке $\left\{(2 k+1) / 2^{j+1}: j, k \in \mathbb{Z}\right\}$.

Д о к а з а т е л ь с т в о. Покажем, что система $\left\{\psi_{I, 0, k}\right\}_{k \in \mathbb{Z}}$ является интерполяционной на сетке $\{l+1 / 2\}_{l \in \mathbb{Z}}$. Действительно, если преобразование Фурье определено формулой (13), то функция $\psi_{I}$ имеет вид

$$
\psi_{I}(x)=\sum_{\nu \in \mathbb{Z}}(-1)^{\nu} \bar{h}_{I, 1-\nu} \varphi_{I, 1, \nu}(x) .
$$

Так как все четные коэффициенты, кроме нулевого, равны нулю, а система функций $\left\{\varphi_{I, 1, k}(x)\right\}$ является интерполяционной на сетке $l / 2, l \in \mathbb{Z}$, то

$$
\psi_{I}(x)=\varphi_{I}(2 x-1)+\sqrt{2} \sum_{\nu \in \mathbb{Z}} \bar{h}_{I, 1-2 \nu} \varphi_{I}(2 x-2 \nu) .
$$

Тогда в точках вида $l+\frac{1}{2}$ будет выполнено

$$
\psi_{I}\left(l+\frac{1}{2}\right)=\varphi_{I}\left(2\left(l+\frac{1}{2}\right)-1\right)+\sqrt{2} \sum_{\nu \in \mathbb{Z}} \bar{h}_{I, 1-2 \nu} \varphi_{I}\left(2\left(l+\frac{1}{2}\right)-2 \nu\right)=\delta_{l, 0} .
$$

Следовательно, система $\left\{\psi_{I}(x-k)\right\}_{k \in \mathbb{Z}}$ является интерполяционной на сетке $\{l+1 / 2\}_{l \in \mathbb{Z}}$, а тогда и система сжатий этих функций $\left\{\psi_{I}\left(2^{j} x-k\right)\right\}_{k \in \mathbb{Z}}$ будет интерполяционной на сетке $\left\{(2 l+1) / 2^{j+1}\right\}_{l \in \mathbb{Z}}$.

Ортогональность системы $\left\{\psi_{I, j, k}\right\}_{j, k \in \mathbb{Z}}$ следует из построения, которое аналогично построению классических ортогональных всплесков по известным ортогональным масштабирующим функциям.

П р и м е р ы.

1. Пусть $m(\omega)$ - маска Мейера. Тогда приведенный способ построения приведет к масштабирующей функции, полученной способом (2).

2. Известно (см. $[4$, гл. 6]), что не существует базисов всплесков с компактным носителем, которые являются одновременно ортогональными и интерполяционными. Рассмотрим в 
качестве $m(\omega)$ маски Добеши $m(\omega)=\sum_{k=0}^{N} h_{k} e^{2 \pi i k \omega}$. Они являются тригонометрическими полиномами, которые удовлетворяют условиям теоремы 1, поэтому построенные с их помощью маски

$$
m_{I}(\omega)=\left|\sum_{k=0}^{N} h_{k} e^{2 \pi i k \omega}\right|^{2}+i \operatorname{sign}(\sin 2 \pi \omega)\left|\sum_{k=0}^{N} h_{k} e^{2 \pi i k \omega} \sum_{l=0}^{N} h_{l} e^{2 \pi i(l+1 / 2) \omega}\right|
$$

позволяют получить интерполяционно-ортогональные системы масштабирующих функций. Отметим, что полученные таким образом масштабирующие функции, в отличие от исходных, не будут иметь компактного носителя, так как маска $m_{I}(\omega)$ здесь не является тригонометрическим полиномом. Тем не менее такие маски имеют более простой вид, чем в общем случае, когда вместо конечных сумм в формуле (14) использовались бы бесконечные ряды.

\section{4. Быстрые дискретные всплеск-преобразования}

Так как свойство ортогональности в случае интерполяционно-ортогональных базисов сохраняется, то для полученных базисов, как и в классическом случае, можно использовать быстрые алгоритмы прямых и обратных дискретных всплеск-преобразований для обработки сигналов. При этом вычисляются коэффициенты разложения функции по базисам масштабирующих функций при малых $j$ по известным коэффициентам разложения функции по базисам масштабирующих функций при больших $j$.

Пусть нам известно разложение функции по базисам $V_{j}^{I}$ и $W_{j}^{I}$ :

$$
\operatorname{Pr}_{V_{j}^{I}} f(x)=\sum_{k \in \mathbb{Z}} c_{j, k}^{I} \varphi_{I, j, k}(x) ; \quad \operatorname{Pr}_{W_{j}^{I}} f(x)=\sum_{k \in \mathbb{Z}} d_{j, k}^{I} \psi_{I, j, k}(x) .
$$

Так как $V_{j+1}^{I}=V_{j}^{I} \oplus W_{j}^{I}$, то

$$
\sum_{k \in \mathbb{Z}} c_{j+1, k}^{I} \varphi_{I, j+1, k}(x)=\sum_{k \in \mathbb{Z}} c_{j, k}^{I} \varphi_{I, j, k}(x)+\sum_{k \in \mathbb{Z}} d_{j, k}^{I} \psi_{I, j, k}(x) .
$$

Скалярно домножая (15) последовательно на $\varphi_{I, j+1, l}(x), \varphi_{I, j, l}(x), \psi_{I, j, l}(x)$, получим, что

$$
\begin{gathered}
c_{j+1, l}^{I}=\sum_{k \in \mathbb{Z}} c_{j, k}^{I} h_{I, l-2 k}+\sum_{k \in \mathbb{Z}} d_{j, k}^{I} h_{I, \psi, l-2 k} ; \\
c_{j, l}^{I}=\sum_{k \in \mathbb{Z}} c_{j+1, k}^{I} \overline{h_{I, 2 l-k}} ; \quad d_{j, l}^{I}=\sum_{k \in \mathbb{Z}} c_{j+1, k}^{I} \overline{h_{I, \psi, 2 l-k}} .
\end{gathered}
$$

Схематично это можно изобразить в виде прямой и обратной пирамидальных схем:

$$
\begin{aligned}
& \begin{array}{c}
c_{j, k}^{I} \longrightarrow c_{j-1, k}^{I} \longrightarrow \\
\\
\end{array} \\
& \begin{array}{lllll}
c_{j-1, k}^{I} & \longrightarrow & c_{j, k}^{I} & \longrightarrow & \\
d_{j-1, k}^{I} & & d_{j, k}^{I} & &
\end{array}
\end{aligned}
$$

\section{СПИСОК ЛИТЕРАТУРЫ}

1. Meyer Y. Wavelets and applications // Proc. Internat. Conf. on Wavelets (May 1989, Marseille, France). Paris: Masson, 1992. 450 p.

2. Mallat S. Muultiresolution approximation and wavelets // Trans. Amer. Math. Soc. 1989. Vol. 315, no. 1. P. $69-88$. doi: $10.2307 / 2001373$. 
3. Субботин Ю.Н., Черных Н.И. Интерполяционно-ортогональные системы всплесков // Тр. Ин-та математики и механики УрО РАН. 2008. Т. 14, № 3. С. 153-161.

4. И. Добеши Десять лекций по вейвлетам. Ижевск: "НИЦ Регулярная и хаотическая динамика", 2001. $464 \mathrm{c}$.

Поступила 26.08.2020

После доработки 2.11.2020

Плещева Екатерина Александровна

Принята к публикации 9.11.2020

канд. физ.-мат. наук,

старший науч. сотрудник

Институт математики и механики им. Н. Н. Красовского УрО РАН;

доцент кафедры математического анализа

Уральский федеральный университет

г. Екатеринбург

e-mail: eplescheva@gmail.com

\section{REFERENCES}

1. Meyer Y. Wavelets and applications. Proc. Internat. Conf. on Wavelets, May 1989, Marseille, France. Paris: Masson, 1992, 450 p. ISBN: 0387545166.

2. Mallat S. Multiresolution approximation and wavelets. Trans. Amer. Math. Soc., 1989, vol. 315, no. 1, pp. 69-88. doi: 10.2307/2001373.

3. Subbotin Yu.N., Chernykh N.I. Interpolating-orthogonal wavelet systems. Proc. Steklov Inst. Math. (Suppl.), 2009, vol. 264, suppl. 1, pp. S107-S115. doi: 10.1134/S0081543809050083.

4. Daubechies I. Ten lectures on wavelets. SIAM, 1992, 377 p. ISBN: 0898712742 . Translated to Russian under the title Desyat' lektsii po veivletam, Izhevsk: "NITs Regulyarnaya i khaoticheskaya dinamika", 2001,464 p.

Received August 26, 2020

Revised November 2, 2020

Accepted November 9, 2020

Funding Agency: This study is a part of the research carried out at the Ural Mathematical Center.

Ekaterina Aleksandrovna Pleshcheva, Cand. Sci. (Phys.-Math.), Krasovskii Institute of Mathematics and Mechanics of the Ural Branch of the Russian Academy of Sciences, Yekaterinburg, 620108 Russia; Ural Federal University, Yekaterinburg, 620000 Russia, e-mail: eplescheva@gmail.com .

Cite this article as: E. A. Pleshcheva. Interpolating orthogonal bases of an MRA and wavelets, Trudy Instituta Matematiki i Mekhaniki UrO RAN, 2020, vol. 26, no. 4, pp. 224-233. 